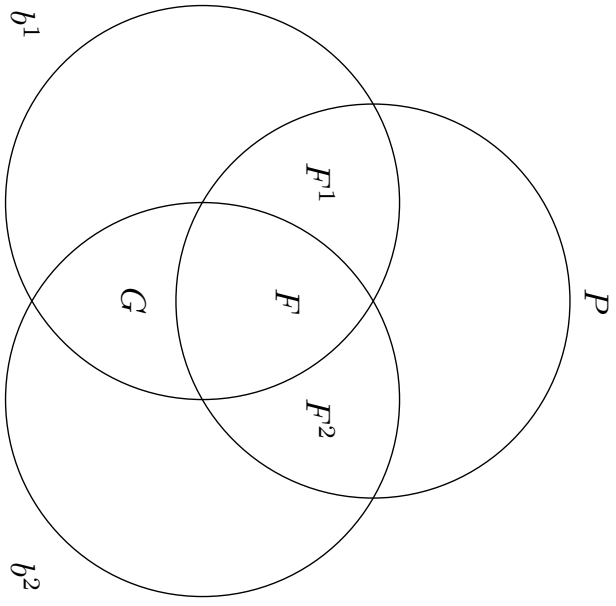




\title{
Whitham Deformations and the Space of Harmonic Tori in $\mathbb{S}^{3}$
}

\author{
Emma Carberry and Ross Ogilvie
}

\begin{abstract}
In this paper we investigate the space of harmonic maps from a 2-torus to $\mathbb{S}^{3}$ using the spectral curve correspondence and Whitham deformations. In an open and dense subset of a parameter space we find that the space of harmonic maps is smooth and has dimension two. We also show that the points that correspond to minimal tori (conformal harmonic maps) are either smooth points of dimension two or singular.
\end{abstract}

\section{Introduction}

In this paper we investigate the space of harmonic maps from a 2-torus to $\mathbb{S}^{3}$. From work of Hitchin[Hit90], each harmonic map uniquely corresponds to spectral data $\left(\Sigma, \Theta^{1}, \Theta^{2}, E\right)$ consisting of a real hyperelliptic curve $\Sigma$, a pair of meromorphic differentials $\Theta^{1}, \Theta^{2}$, and a quaternionic line bundle $E$, which satisfy a litany of conditions (this correspondence excludes the case of conformal harmonic maps into a 2-sphere). In particular, there are constraints on the periods of the differentials. This correspondence makes it possible to construct a parameter space for the spectral triples $\left(\Sigma, \Theta^{1}, \Theta^{2}\right)$. Letting $\mathcal{M}_{g}$ be the space of spectral triples $\left(\Sigma, \Theta^{1}, \Theta^{2}\right)$ with a spectral curve $\Sigma$ of genus $g$, the conditions that spectral data must satisfy allow us to parameterise spectral triples by polynomials of a fixed degree. We can then identify $\mathcal{M}_{g}$ as a subset of this parameter space. It is possible then to characterise the tangent vectors to $\mathcal{M}_{g}$ by equations (3.3) and (3.8), using Whitham deformations. At every point $p$ of an open and dense subset $\mathcal{U}_{a b}$ of the space of polynomials, where some additional assumptions hold, we find all solutions to these equations to demonstrate that $T_{p} \mathcal{M}_{g}$ is two-dimensional (Lemmata 5.6 and 5.7). Having established that the dimension is constant, it naturally follows that $\mathcal{M}_{g} \cap \mathcal{U}_{a b}$ is a manifold (Theorem 5.8). A similar analysis at the conformal harmonic maps show that they are isolated in $\mathcal{M}_{g}$ or smooth points of dimension two.

The essential methodology is to describe the deformations that preserve the periods of differentials, the so called Whitham deformations. This method was 
first discovered for the Korteweg-de Vries equation [FFM80, LL83], before being developed generally for other integrable systems [Kri95, GKM $\left.{ }^{+} 95\right]$. It is difficult to describe the curves with a constraint on their periods directly, due to the Schottky problem [Gru12, Shi86]. Whitham deformations are therefore a powerful technique to extract local information. The form of Whitham deformations used here resemble their application in the theory of constant mean curvature surfaces, with similar goals. In [KSS15] it was shown that the space of equivariant CMC tori in $\mathbb{S}^{3}$ is a connected infinite graph. [CS16a] uses Whitham deformations to show that for each $H>0$ and each fixed genus of the spectral curve, spectral triples of tori of constant mean curvature $H$ in $\mathbb{S}^{3}$ are dense amongst those of CMC planes. When the ambient space is $\mathbb{R}^{3}$, the tori are no longer dense and [CS16b] gives a partial description of their closure.

The reason to concentrate on spectral triples and ignore the line bundle is that deformations of the line bundle are well understood. Given a spectral datum, if one holds $\Sigma$ fixed then it is not possible to deform the differentials, because they must have integral periods, but it is possible to deform $E$. These are isospectral deformations and the space of possible $E$ are described by a real $g$-dimensional torus in $\operatorname{Pic}^{g+1}(\Sigma)$. Thus we focus on non-isospectral deformations, which are deformations of $\Sigma$ that preserve the integrality conditions of the differentials.

In our preliminaries (Section 2) we lay out the conditions that spectral data must satisfy and show how these conditions may be used to represent spectral triples $\left(\Sigma, \Theta^{1}, \Theta^{2}\right)$ as a triple of polynomials $\left(P, b^{1}, b^{2}\right)$. We describe an open and dense subspace $\mathcal{U}$ of the space of polynomials that partially satisfy the conditions to be spectral data. Hence $\mathcal{M}_{g}$ lies in $\mathcal{U}$. If we take a path in $\mathcal{M}_{g}$ parameterised by $t$, then because the periods of the differentials are integral, they are constant. Thus the $t$-derivative of each differential is exact, and yields a meromorphic function. Just as we characterised the differentials by a polynomial, so too can this pair of meromorphic functions be described by polynomials $\left(\hat{c}^{1}, \hat{c}^{2}\right)$. The central result of Section 3 is Lemma 3.4, which shows each tangent vector to $\mathcal{M}_{g}$ is associated to a unique pair of polynomials $\left(\hat{c}^{1}, \hat{c}^{2}\right)$ and shows that this pair must satisfy a certain restrictive relationship (3.14).

A standalone interlude, Section 4, gives elementary but useful variations of Bézout's identity adapted for the types of polynomial equations found in this field of study. With these tools, it is then possible to establish the main result of the paper in Section 5. This is the result that $\mathcal{M}_{g} \cap \mathcal{U}_{a b}$ is a smooth twodimensional manifold, for $\mathcal{U}_{a b}$ an open and dense subset of the parameter space. Section 6 gives a similar result, that generically conformal harmonic maps (i.e. minimal surfaces) are smooth points of $M_{g}$. 


\section{Preliminaries}

Hitchin [Hit90] investigated harmonic maps from a torus to the 3-sphere and characterised them in terms of a spectral curve construction. The spectral data corresponding to a harmonic map $T^{2} \rightarrow \mathbb{S}^{3}$ consists of a tuple $\left(\Sigma, \Theta^{1}, \Theta^{2}, E\right)$. $\Sigma$ is called the spectral curve and is a real hyperelliptic curve over $\mathbb{C P}^{1}$ with a pair of meromorphic differentials $\Theta^{1}, \Theta^{2}$ and line bundle $E$. Certain geometric features of a harmonic map may be discerned from properties of spectral data. For example, a harmonic map is conformal (and therefore minimal) exactly when the spectral curve is branched over 0. Theorems of Hitchin [Hit90, Theorems 8.1, 8.20] provide a correspondence between harmonic maps from the torus to $\mathbb{S}^{3}$ and tuples $\left(\Sigma, \Theta^{1}, \Theta^{2}, E\right)$ satisfying properties (P.1)-(P.10). The harmonic map is uniquely determined by its spectral data, up to the action of $\mathrm{SO}(4)$ on $\mathbb{S}^{3}$. We shall now enumerate those properties.

Suppose that $\Sigma$ has genus $g$ and is described by $\eta^{2}=P(\zeta)$ in the total space of $\pi: \mathcal{O}(g+1) \rightarrow \mathbb{C P}^{1}$ with $P(\zeta)$ a section of $\mathcal{O}(2 g+2)$. This curve is hyperelliptic with involution $\sigma(\zeta, \eta)=(\zeta,-\eta)$. A spectral curve has the following properties.

(P.1) Real curve: $P(\zeta)$ is a real section of $\mathcal{O}(2 g+2)$.

(P.2) No real zeroes: $P(\zeta)$ has no zeroes on the unit circle $\mathbb{S}^{1} \subset \mathbb{C P}^{1}$.

(P.3) Simple zeroes: $P(\zeta)$ has only simple zeroes over $\zeta=0, \infty$.

Assumption: In this paper we consider only nonsingular spectral curves. This means $P(\zeta)$ may only have simple roots.

Sections of $\mathcal{O}(k)$ can be identified with polynomials of degree at most $k$. We use the real structure on the line bundle $\mathcal{O}(k)$ given by

$$
\rho_{k}:(\zeta, \eta) \mapsto\left(\bar{\zeta}^{-1}, \bar{\zeta}^{-k} \bar{\eta}\right)
$$

Definition 2.1. Let $\mathcal{P}^{k}$ be the space of polynomials of degree at most $k$. We define the real polynomials $\mathcal{P}_{\mathbb{R}}^{k}$ to be

$$
\mathcal{P}_{\mathbb{R}}^{k}=\left\{q_{0}+\cdots+q_{k} \zeta^{k} \in \mathcal{P}^{k} \mid q_{i}=\overline{q_{k-i}} \text { for all } 0 \leq i \leq k\right\} .
$$

$\mathcal{P}_{\mathbb{R}}^{k}$ is a real vector space of dimension $k+1$.

The polynomials in $\mathcal{P}_{\mathbb{R}}^{k}$ are those that correspond to sections invariant under pullback by the real involution $\rho_{k}$. Every root of a real polynomial must either lie on the unit circle or come in a conjugate-inverse pair. Identifying $P$ with a polynomial in $\mathcal{P}_{\mathbb{R}}^{2 g+2}$, we fix the following scaling of $P$,

$$
P(\zeta)=\prod_{i=0}^{g}\left(\zeta-\alpha_{i}\right)\left(1-\bar{\alpha}_{i} \zeta\right)
$$


for $\alpha_{0}, \ldots, \alpha_{g}$ in the unit disc. Roots on the unit circle are excluded by (P.2). A nice feature of this scaling is that it is well-behaved if one branch point is zero; the corresponding factor becomes $\zeta$. A hyperelliptic curve is determined by its branch points in $\mathbb{C P}^{1}$, uniquely up to automorphism of $\mathbb{C P}^{1}$. Since we have distinguished the points 0,1 , and -1 we have fixed the automorphism, and so every spectral curve corresponds to a unique polynomial of the form (2.2).

Each differential $\Theta^{1}, \Theta^{2}$ must satisfy the following conditions.

(P.4) Poles: The differentials have residue-free double poles at $\pi^{-1}\{0, \infty\}$ but are otherwise holomorphic.

(P.5) Symmetry: The differentials satisfy $\sigma^{*} \Theta^{i}=-\Theta^{i}$.

(P.6) Reality: The differentials satisfy $\rho_{g+3}^{*} \Theta^{i}=-\bar{\Theta}^{i}$.

(P.7) Linear independence: The principal parts of the differentials $\Theta^{1}$ and $\Theta^{2}$ are linearly independent over $\mathbb{R}$.

Every curve satisfying (P.1)-(P.3) has differentials that satisfy (P.4)-(P.6), necessarily of the form

$$
\Theta=b(\zeta) \frac{d \zeta}{\zeta^{2} \eta}=\left(b_{0}+b_{1} \zeta+\cdots+b_{g+3} \zeta^{g+3}\right) \frac{d \zeta}{\zeta^{2} \eta},
$$

for some $b(\zeta) \in \mathcal{P}_{\mathbb{R}}^{g+3}$, by [Mir95, Prop III.1.10]. If $\Sigma$ does not have a branch point at 0 , then the residue of $\Theta$ is

$$
\underset{\zeta=0}{\operatorname{res}} \Theta=b_{1}-\frac{1}{2} \frac{P_{1}}{P_{0}} b_{0},
$$

where subscripts denote coefficients of the polynomials. This quantity must therefore vanish by (P.4). On the other hand, if $P_{0}$ is zero then the same condition forces $b_{0}=0$. In light of this, we may rephrase equation (2.4),

$$
P_{1} b_{0}-2 P_{0} b_{1}=0 .
$$

The differentials belonging to spectral data however have properties further than (P.4)-(P.7), which are 'hard' to satisfy.

(P.8) Periods: The periods of the differentials $\Theta^{1}$ and $\Theta^{2}$ lie in $2 \pi i \mathbb{Z}$.

(P.9) Closing conditions: Let $\gamma_{+}$be a path in $\Sigma$ connecting the two points of $\pi^{-1}(1)=\left\{\xi_{1}, \sigma\left(\xi_{1}\right)\right\}$ and likewise let $\gamma_{-}$connect the two points $\left\{\xi_{-1}, \sigma\left(\xi_{-1}\right)\right\}$ over -1 . Then

$$
\int_{\gamma_{+}} \Theta^{i} \in 2 \pi i \mathbb{Z}, \quad \text { and } \int_{\gamma_{-}} \Theta^{i} \in 2 \pi i \mathbb{Z}
$$


Finally, the line bundle of the spectral data has the following property.

(P.10) Quaternionic: $E^{*}$ is a line bundle of degree $g+1$ that is quaternionic with respect to the involution $\rho_{g+1} \circ \sigma$.

If the choice of curve and differentials is fixed, one is free to choose $E$ subject only to (P.10). There are many such choices; they form a real $g$ dimensional torus in the Jacobian of $\Sigma$. Variations of $E$ alone are called isospectral deformations. Conversely, if we have a triple $\left(\Sigma, \Theta^{1}, \Theta^{2}\right)$ satisfying the above conditions, then there always exists such a line bundle $E$ completing the tuple. Thus we focus our attention on the problem of deforming the spectral triple $\left(\Sigma, \Theta^{1}, \Theta^{2}\right)$; so called non-isospectral deformations.

We have seen that any spectral triple $\left(\Sigma, \Theta^{1}, \Theta^{2}\right)$ may be described in terms of polynomials $\left(P, b^{1}, b^{2}\right) \in \mathcal{P}_{\mathbb{R}}^{2 g+2} \times \mathcal{P}_{\mathbb{R}}^{g+3} \times \mathcal{P}_{\mathbb{R}}^{g+3}$.

Definition 2.6. We call $\mathcal{M}_{g}$ the space of spectral triples $\left(\Sigma, \Theta^{1}, \Theta^{2}\right)$ satisfying conditions (P.1)-(P.9) and such that $\Sigma$ has genus $g$ and is nonsingular.

Let $\mathcal{U}$ be the following open and dense subset of $\mathcal{P}_{\mathbb{R}}^{2 g+2} \times \mathcal{P}_{\mathbb{R}}^{g+3} \times \mathcal{P}_{\mathbb{R}}^{g+3}$. If $\left(P, b^{1}, b^{2}\right)$ is a point of $\mathcal{U}$ then $P$ has no zeroes on the unit circle (cf. (P.2)) and only simple zeroes elsewhere (cf. (P.3) and $\Sigma$ nonsingular). The polynomials $b^{i}$ have at most a simple root at $\zeta=0(c f .(P .4))$.

We view $\mathcal{M}_{g}$ as a subset of $\mathcal{U}$ using the above correspondence between spectral triples and polynomials.

A comment about notation. A polynomial with a circumflex (hat) will be shown to have a factor of $\zeta^{2}-1$, and a tilde will indicate that common factors have been removed, cf. (3.10). We shall use a dash to denote differentiation with respect to $\zeta$ and a dot for differentiation with respect to $t$ evaluated at $t=0$. When giving the solutions to equations we will use bold to signify a particular solution, which may or may not be unique. Given a tuple of polynomials, such as $(X, Y)$, we also give their degrees as a tuple, e.g. $(x, y)$ for $x=\operatorname{deg} X$ and $y=\operatorname{deg} Y$. Finally, we shall use $i$ and $j$ for indices ranging over $\{1,2\}$, with the understanding that they are not equal.

\section{Deformations of Harmonic Maps}

In this section we lay out the properties of infinitesimal deformations of spectral triples $\left(\Sigma, \Theta^{1}, \Theta^{2}\right)$ within the space $\mathcal{M}_{g}$. A deformation of spectral data is a path $\ell:(-\epsilon, \epsilon) \rightarrow \mathcal{M}_{g}, t \mapsto\left(P(t, \zeta), b^{1}(t, \zeta), b^{2}(t, \zeta)\right)$. An infinitesimal deformation is the tangent vector of such a curve at $t=0$. Denote $\Sigma=\Sigma(0)$ and likewise $b^{i}(\zeta)=b^{i}(0, \zeta)$. More generally, omission of the parameter $t$ will correspond to evaluation at the point $t=0$. 
Consider the $t$-derivative of $\Theta^{i}$. The periods of $\Theta^{i}$ are constant in $t$ by (P.8), so $\dot{\Theta}^{i}$ is exact. Write $\dot{\Theta}^{i}=d \theta^{i}$, for $\theta^{i}$ a meromorphic function on $\Sigma$. In order to write $\theta^{i}$ analogously to how we wrote $\Theta^{i}$ in (2.3) we need to know how a function may acquire additional poles when it is differentiated with respect to $t$. This must be handled delicately; consider the example of $f(t)(z)=\left(z^{n}+t\right) /(z-t)^{n}$, where $f(0) \equiv 1$ but $\dot{f}$ has a pole of order $n$ at $z=0$.

Lemma 3.1. Consider a smooth family of hyperelliptic curves $\Sigma(t)$ and a smooth family of meromorphic functions $f(t)$, for $t \in(-\epsilon, \epsilon)$. Fix a point $p \in \Sigma(0)$ that lies over $\beta \in \mathbb{C} P^{1}$.

Suppose $p$ is a not ramification point. If $f(t)$ is holomorphic for every $t$ then $\dot{f}$ is holomorphic. If $(\zeta-\beta)^{n} f(t)$ is holomorphic for every $t$ then $\dot{f}$ may have a pole of at most order $n$ at $p$.

Suppose that $p$ is a ramification point. If $f(t)$ is holomorphic for every $t$ then $\dot{f}$ may have at worst a simple pole at $p$.

Proof. Let us consider the unramified case first. Near $p$ we may write our function $f(t)(\zeta)=u(t, x, y)+i v(t, x, y)$ for $\zeta=x+i y$. As $f(t)$ is holomorphic we have the Cauchy-Riemann equations $\partial_{x} u=\partial_{y} v$ and $\partial_{y} u=-i \partial_{x} v$. Because $f(t)$ is a smooth function we may differentiate by $t$ and interchange the order, yielding $\partial_{x} \partial_{t} u=\partial_{y} \partial_{t} v$ and $\partial_{y} \partial_{t} u=-i \partial_{x} \partial_{t} v$. This shows that $\dot{f}$ is holomorphic too. The second part then follows because the left hand side of

$$
\frac{d}{d t}(\zeta-\beta)^{n} f=(\zeta-\beta)^{n} \partial_{t} f
$$

is now known to be holomorphic.

We move now to the ramified case. Let $\alpha(t)$ be a branch point of $\Sigma(t)$ such that $\alpha(0)=\beta$. Note that there is a unique choice. On $\Sigma(t)$ take $\xi(t)^{2}=\zeta-\alpha(t)$ as local coordinate, so we may compute

$$
\frac{d}{d t} f(t)(\xi)=\frac{\partial f}{\partial t}-\frac{1}{2} \dot{\alpha} \xi^{-1} \frac{\partial f}{\partial \xi} .
$$

If $f(t)$ was holomorphic, then we see that $\dot{f}$ may have a simple pole.

As $\Theta^{i}$ has double poles without residues over $\zeta=0$ and $\infty$, it follows that $\theta^{i}$ may have simple poles at the roots of $P$ in $\mathbb{C}^{\times}$. If the curve $\Sigma$ is unbranched over $\zeta=0$ and $\infty$, then $\theta^{i}$ has at worst simple poles there. If the curve $\Sigma$ is branched over $\zeta=0$, then by (2.5) there exists a root $\beta(t)$ of $\Theta^{i}(t)$ such that $\zeta^{2}(\zeta-\beta(t))^{-1} \Theta^{i}(t)$ is holomorphic near $\zeta=0$ for all $t$ and $\beta(0)=0$. Differentiating gives

$$
\dot{\Theta^{i}}=\dot{\beta} \zeta^{-1} \Theta^{i}+\left.\zeta^{-1} \frac{d}{d t} \zeta^{2}(\zeta-\beta(t))^{-1} \Theta^{i}(t)\right|_{t=0}
$$


from which we can see that $\dot{\Theta}^{i}$ has at worst a fourth order pole over $\zeta=0$ in this case. Therefore $\theta^{i}$ may have a triple pole there. Hence $\zeta \eta \theta^{i}$ is holomorphic over $\mathbb{C}$ and for some degree $g+3$ polynomial $\hat{c}^{i}$

$$
\theta^{i}=\frac{1}{\zeta \eta} \hat{c}^{i}(\zeta)
$$

By definition, $\dot{\Theta}^{i}=d \theta^{i}$. This provides equations linking $\dot{b}^{i}$ and $\hat{c}^{i}$,

$$
\dot{P} b^{i}-2 P \dot{b}^{i}=2 P\left(\hat{c}^{i}-\zeta \hat{c}^{i \prime}\right)+P^{\prime} \zeta \hat{c}^{i} .
$$

It is natural to ask to what extent the functions $\theta^{i}$ determine an infinitesimal deformation $\left(\dot{P}, \dot{b}^{1}, \dot{b}^{2}\right)$. In Lemma 3.4 we will show that $\theta^{1}$ and $\theta^{2}$ are uniquely determined by an infinitesimal deformation. Thus we may use these functions to identify the space of infinitesimal deformations that preserve periods with a subset of $\mathcal{P}^{g+3} \times \mathcal{P}^{g+3}$. The converse question is more difficult and is addressed in Section 5 .

Lemma 3.4. Given a point $\left(P, b^{1}, b^{2}\right)$ of $\mathcal{M}_{g}$ for which there are deformations, the polynomials $\left(\hat{c}^{1}, \hat{c}^{2}\right)$ are determined uniquely by a tangent vector $\left(\dot{P}, \dot{b}^{1}, \dot{b}^{2}\right)$ to $\mathcal{M}_{g}$.

Proof. Since the equations (3.3) are linear in the components of the tangent vector, we need only demonstrate that the zero tangent vector uniquely corresponds to $\hat{c}^{i}=0$. For the zero tangent vector,

$$
0=2 P\left(\hat{c}^{i}-\zeta \hat{c}^{i \prime}\right)+P^{\prime} \zeta \hat{c}^{i} .
$$

The polynomial $P$ is either of the form $L$ or $\zeta L$, where $L$ has only roots in $\mathbb{C}^{\times}$. $L$ has degree either $2 g+2$ or $2 g$ respectively. The assumption of a nonsingular spectral curve requires that $P$ and $P^{\prime}$ have no common factors, hence evaluation of (3.5) at any root $\alpha$ of $L$ shows that $P^{\prime}(\alpha) \alpha \hat{c}^{i}(\alpha)=0$, and hence $\alpha$ is a root of $\hat{c}^{i}$. This shows that $L$ divides $\hat{c}^{i}$, and for $g \geq 4$ the inequality $\operatorname{deg} L \geq 2 g \geq g+4$ is sufficient to show that $\hat{c}^{i}$ is the zero polynomial, as it is a degree $g+3$ polynomial that is divisible by a polynomial of greater degree. To handle the remaining cases, $g<4$, we substitute in this factorisation of $\hat{c}^{i}$ and then remove the factor of $L$,

$$
0=L\left[2 \frac{P}{L} \frac{\hat{c}^{i}}{L}-2 \zeta \frac{P}{L}\left(\frac{\hat{c}^{i}}{L}\right)^{\prime}+\zeta\left(\frac{P}{L}\right)^{\prime} \frac{\hat{c}^{i}}{L}\right]-\zeta L^{\prime} \frac{P}{L} \frac{\hat{c}^{i}}{L} .
$$

Again, this shows that $L$ divides $\hat{c}^{i} / L$. If $\operatorname{deg} L=2 g+2$, this shows that $\hat{c}^{i}$ is divisible by a polynomial of degree $4 g+4$, and so must be zero for any $g$. If $\operatorname{deg} L=2 g$, then we have only shown that $\hat{c}^{i}$ vanishes for $g \geq 2$. We treat the two remaining cases, $\operatorname{deg} L=2 g$ and $g=0$ or 1, individually. 
If $\operatorname{deg} L=2 g$ and $g=1$, then $\hat{c}^{i}$ is a scalar multiple of $L^{2}$. Let $\hat{c}^{i}=a L^{2}$ and equation (3.5) simplifies to

$$
0=2 a L^{3}\left(3 L-2 \zeta L^{\prime}\right),
$$

which forces $a=0$. For the second case if $g=0$ then $P=\zeta$ and $\hat{c}^{i}$ is a cubic polynomial. After removing the factor of $\zeta$,

$$
0=2\left(\hat{c}^{i}-\zeta \hat{c}^{i \prime}\right)+\hat{c}^{i}=3 \hat{c}_{0}^{i}+\hat{c}_{1}^{i} \zeta-\hat{c}_{2}^{i} \zeta^{2}-3 \hat{c}_{3}^{i} \zeta^{3},
$$

which again shows that $\hat{c}^{i}$ is zero. Hence, the polynomials $\hat{c}^{i}$ are uniquely determined by a tangent vector $\left(\dot{P}, \dot{b}^{1}, \dot{b}^{2}\right)$ to $\mathcal{M}_{g}$ as claimed.

Continuing our line of inquiry into the properties of $\hat{c}^{i}$, recall our supposition that we are at a point $\left(P, b^{1}, b^{2}\right)$ of $\mathcal{M}_{g}$ that admits a deformation, from which we have defined polynomials $\hat{c}^{1}$ and $\hat{c}^{2}$ and derived the pair of equations (3.3). Note that the two equations (3.3) for $i=1,2$ are not independent of one another, for they both contain $P$ and its derivatives. If we multiply the equations by $\hat{c}^{2}$ and $\hat{c}^{1}$ respectively and take the difference, we observe

$$
\dot{P}\left(b^{1} \hat{c}^{2}-b^{2} \hat{c}^{1}\right)=2 P\left(\dot{b}^{1} \hat{c}^{2}-\dot{b}^{2} \hat{c}^{1}-\zeta \hat{c}^{1 \prime} \hat{c}^{2}+\zeta \hat{c}^{2 \prime} \hat{c}^{1}\right) .
$$

We will prove that $b^{1} \hat{c}^{2}-b^{2} \hat{c}^{1}$ is divisible by $P$ by showing that it vanishes at every root of $P$. If $\alpha$ is a root of $P$ and not a root of $\dot{P}$, we see it is a root of $b^{1} \hat{c}^{2}-b^{2} \hat{c}^{1}$ immediately from (3.6). Suppose then that $P$ and $\dot{P}$ have a common root $\alpha$. If $\alpha=0$, then we know from (2.5) that $b_{0}^{i}=0$ and so $\zeta$ divides $b^{i}$. If $\alpha \neq 0$, from (3.3) we have that

$$
\begin{aligned}
\dot{P}(\alpha) b^{i}(\alpha) & =2 P(\alpha)\left(\dot{b}^{i}(\alpha)+\hat{c}^{i}(\alpha)-\alpha \hat{c}^{i \prime}(\alpha)\right)+P^{\prime}(\alpha) \alpha \hat{c}^{i}(\alpha) \\
0 & =0+P^{\prime}(\alpha) \alpha \hat{c}^{i}(\alpha)
\end{aligned}
$$

But the assumption that the spectral curve is nonsingular forces $P^{\prime}(\alpha) \neq 0$. Thus we may conclude that $\hat{c}^{i}(\alpha)=0$. Hence $P$ divides $b^{1} \hat{c}^{2}-b^{2} \hat{c}^{1}$ and there is some polynomial $\hat{Q}$ of degree at most four such that

$$
b^{1} \hat{c}^{2}-b^{2} \hat{c}^{1}=\hat{Q} P .
$$

Thus far we have only placed two conditions on the points along the deformation. First, that it must preserve the integral periods of $\Theta^{1}$ and $\Theta^{2}$, which allowed us to produce well-defined meromorphic functions $\theta^{i}$. And second that the differentials must have double poles over $\zeta=0$ and $\infty$ (Property (P.4)), which allowed us to write $\theta^{i}$ as the quotient of a polynomial $\hat{c}^{i}$ by $\zeta \eta$.

Applying (P.6) forces the polynomials $\hat{c}^{i}$ to be imaginary (that is, $i \hat{c}^{i} \in \mathcal{P}_{\mathbb{R}}^{g+3}$ ). Next, consider the closing condition (P.9). Differentiating

$$
0=\left.\frac{d}{d t}\right|_{t=0} \int_{\gamma_{+}} \Theta^{i}=\int_{\gamma_{+}} d \theta^{i}=\theta^{i}\left(\sigma\left(\xi_{1}\right)\right)-\theta^{i}\left(\xi_{1}\right)
$$


where $\xi_{1}$ is a point in $\Sigma$ over $\zeta=1$. But

$$
\theta^{i}\left(\sigma\left(\xi_{1}\right)\right)=\sigma^{*} \theta^{i}\left(\xi_{1}\right)=-\theta^{i}\left(\xi_{1}\right) .
$$

Thus $\hat{c}^{i}$ has a root at $\zeta=1$. The same reasoning applied to $\gamma_{-}$leads to a root at $\zeta=-1$. Therefore let $\hat{c}^{i}(\zeta)=\left(\zeta^{2}-1\right) c^{i}(\zeta)$ for some $c^{i} \in \mathcal{P}_{\mathbb{R}}^{g+1}$.

As $\zeta^{2}-1$ is a factor of both polynomials $\hat{c}^{i}$, and $P$ has no zeroes on the unit circle, it follows from (3.7) that $\zeta^{2}-1$ must be a factor of $\hat{Q}$. Define $\hat{Q}=\left(\zeta^{2}-1\right) Q$ to give

$$
b^{1} c^{2}-b^{2} c^{1}=Q P
$$

for some real quadratic polynomial $Q$. The importance of this equation is that it ensures that the solutions to the two equations (3.3) are consistent with one another.

The final condition on the spectral data that we are yet to satisfy is condition (P.4): that the differentials $\Theta^{1}$ and $\Theta^{2}$ are residue free. We shall require $P_{1}(t) b_{0}^{i}(t)-2 P_{0}(t) b_{1}^{i}(t)=0$ to hold at every point of the deformation (from (2.5)). Taking derivatives,

$$
\dot{P}_{1} b_{0}^{i}+P_{1} \dot{b}_{0}^{i}-2 \dot{P}_{0} b_{1}^{i}-2 P_{0} \dot{b}_{1}^{i}=0
$$

holds for any for tangent vector $\left(\dot{P}, \dot{b}^{1}, \dot{b}^{2}\right)$ to $\mathcal{M}_{g}$.

This covers the necessary properties that are shared by all infinitesimal deformations. However, there are further constraints in the special cases where the differentials have common zeroes or zeros at ramification points. In these cases, the polynomial $Q$ also shares a common root. Suppose we have the following common factors at the point $\left(P(0, \zeta), b^{1}(0, \zeta), b^{2}(0, \zeta)\right) \in \mathcal{M}_{g}$ :

$$
\begin{gathered}
\operatorname{gcd}\left(P, b^{1}, b^{2}\right)=F, \\
\operatorname{gcd}\left(P / F, b^{1} / F\right)=F^{1}, \operatorname{gcd}\left(P / F, b^{2} / F\right)=F^{2}, \\
\operatorname{gcd}\left(b^{1} / F F^{1}, b^{2} / F F^{2}\right)=G,
\end{gathered}
$$

where we first find the common factor of all three polynomials, then remove any further factors that the differentials and $P$ share, and then finally remove any remaining factors common to $b^{1}$ and $b^{2}$. An graphic representation of this process is given in Figure 3.1. We write

$$
P=F F^{1} F^{2} \tilde{P}, \quad b^{1}=F F^{1} G \tilde{b}^{1}, \quad b^{2}=F F^{2} G \tilde{b}^{2} .
$$

Because the spectral curve is nonsingular, $P$ has no repeated factors, and so the polynomials $F, F^{1}, F^{2}, \tilde{P}, \tilde{b}^{1}$ and $\tilde{b}^{2}$ are pairwise coprime. The polynomials $b^{1}$ and $b^{2}$ may have higher order roots, so it is not possible to say if $G$ is coprime to $F, F^{1}$ or $F^{2}$. The common factor of $b^{1}$ and $b^{2}$, and therefore any differential on $\Sigma$ satisfying conditions (P.4)-(P.8), is $F G$. We denote the degrees of the polynomials $F, F^{1}, F^{2}$ and $G$ as $d_{F}, d_{1}, d_{2}$ and $d_{G}$ respectively. 


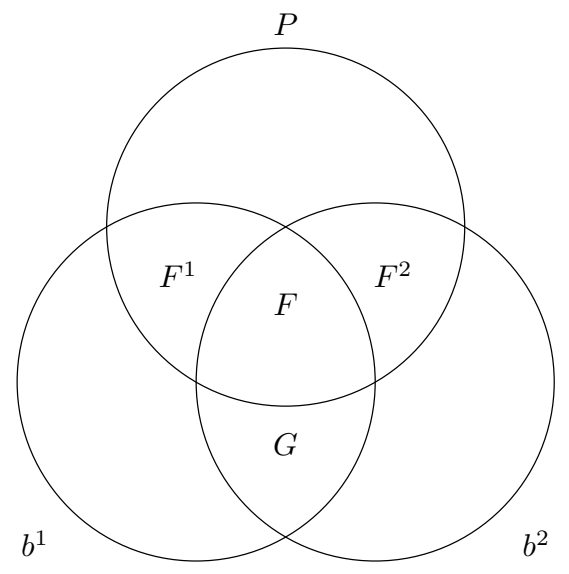

Figure 3.1

The common factors of $P$, $b^{1}$ and $b^{2}$ are represented as overlaps between the three circles.

Inserting these factorisations into (3.3), we observe that

$$
\dot{P} F F^{i} G \tilde{b}^{i}=2 F F^{1} F^{2} \tilde{P}\left(\dot{b}^{i}+\hat{c}^{i}-\zeta \hat{c}^{i \prime}\right)+\zeta\left(\zeta^{2}-1\right) P^{\prime} c^{i} .
$$

Again, by the assumption of that the spectral curve is nonsingular, $P^{\prime}$ does not share any common factors with $P$. Further $P$ has no roots on the unit circle. Hence we see that $F F^{i}$ divides $\zeta c^{i}$.

Assumption: We assume that $P(0) \neq 0$, which corresponds to a nonconformal harmonic map. This assumption will persist until Section 6 , where we deal with the conformal case.

Having made this assumption $\zeta$ is not a factor of $P$ and so cannot be a factor of $F F^{i}$. Therefore $F F^{i}$ divides $c^{i}$. Applying this to (3.8),

$$
F F^{1} G \tilde{b}^{1} c^{2}-F F^{2} G \tilde{b}^{2} c^{1}=Q F F^{1} F^{2} \tilde{P} .
$$

By definition, neither $F$ nor $G$ divide $\tilde{P}$, demonstrating that $F G$ divides $Q$. This provides a bound on the number of coincident roots that are allowed if a deformation is to exist; $Q$ is quadratic so $F G=\operatorname{gcd}\left(b^{1}, b^{2}\right)$ must be degree two or less. Moreover, because all of $P, b^{1}, b^{2}$ are real, and $P$ has no roots on the unit circle, any common roots of the three polynomials must come in conjugate inverse pairs and so the degree of $F$ will always be even.

Assuming that a deformation does exist, $F F^{i}$ must divide $c^{i}$ and $F G$ must divide $Q$. If the polynomials $c^{i}$ or $Q$ did not have the factors indicated by (3.12), then there would be factors on the left hand sides of (3.3) and (3.8) that did not appear on the right hand sides, and this contradiction would preclude the possibility of a solution. Hence equations (3.13) and (3.14) are necessary conditions 
to be able to solve (3.3) and (3.8) respectively. Let us define

$$
c^{i}=F F^{i} \tilde{c}^{i}, \text { and } Q=F G \tilde{Q} .
$$

We can then remove the common factor $F F^{i}$ from (3.3) to arrive at the reduced equations

$$
\dot{P} G \tilde{b}^{i}-2 F^{j} \tilde{P} \dot{b}^{i}=2 F^{j} \tilde{P}\left(\hat{c}^{i}-\zeta \hat{c}^{i \prime}\right)+\zeta\left(\zeta^{2}-1\right) P^{\prime} \tilde{c}^{i},
$$

for $i=1,2$ and $j \neq i$. In the same manner, the $Q$ equation (3.8) reduces to

$$
\begin{aligned}
F F^{1} G \tilde{b}^{1} F F^{2} \tilde{c}^{2}-F F^{2} G \tilde{b}^{2} F F^{1} \tilde{c}^{1} & =F G \tilde{Q} F F^{1} F^{2} \tilde{P} \\
\tilde{b}^{1} \tilde{c}^{2}-\tilde{b}^{2} \tilde{c}^{1} & =\tilde{Q} \tilde{P} .
\end{aligned}
$$

To recap, any tangent vector $\left(\dot{P}, \dot{b}^{1}, \dot{b}^{2}\right)$ to $\mathcal{M}_{g}$ must satisfy (3.9). Each tangent vector gives rise to a pair of polynomials $\left(c^{1}, c^{2}\right) \in \mathcal{P}_{\mathbb{R}}^{g+1} \times \mathcal{P}_{\mathbb{R}}^{g+1}$ through (3.3). The polynomials $\left(c^{1}, c^{2}\right)$ must themselves factorise as per (3.12) and satisfy (3.14) for some real polynomial $\tilde{Q}$ of degree at most 2 . These are necessary and sufficient conditions for a triple of polynomials to be a tangent vector to $\mathcal{M}_{g}$. Under various additional assumptions, Lemmata 5.6, 5.7, and 6.2 find all solutions to these equations.

\section{Bézout Identity}

Equations such as (3.3) and (3.14) are of the form

$$
A X-B Y=C,
$$

to which Bézout's identity for polynomials applies. This section will develop a variant of Bézout's identity adapted to the particulars of our situation. The basic version of Bézout's identity for polynomials asserts that if $\operatorname{gcd}(A, B)=$ 1 , then there is a unique solution $(\mathbf{X}, \mathbf{Y})$ of minimal degree, with $\operatorname{deg} \mathbf{X}<$ $\operatorname{deg} B$ and $\operatorname{deg} \mathbf{Y}<\operatorname{deg} A$. Let us fix $A, B, C$ to be polynomials of degree $a, b, c$ respectively and $d$ to be the degree of $D:=\operatorname{gcd}(A, B)$.

Assumption: Throughout this section, we assume that $\operatorname{gcd}(A, B)$ divides $C$. This is a necessary condition to the solution of (4.1).

Lemma 4.2. There is a unique solution $(\mathbf{X}, \mathbf{Y})$ to (4.1) such that the degree of $\mathbf{X}$ is at most $b-d-1$. Moreover, if $c<a+b-d$ then the degree of $\mathbf{Y}$ is at most $a-d-1$.

Proof. Because we will have need of the specific formula (4.3) we construct the minimal solution by giving a linear system of equations that the coefficients of $\mathbf{X}$ must satisfy and showing that there is a unique solution to this linear system. 
Suppose then that $\mathbf{X}$ is degree $b-d-1$. By dividing (4.1) by the common factor $D=\operatorname{gcd}(A, B)$, consider the equation $(A / D) X-(B / D) Y=C / D$. If $\beta$ is a root of $B / D$ then evaluation yields,

$$
X_{0}+X_{1} \beta+X_{2} \beta^{2}+\cdots+X_{b-d-1} \beta^{b-d-1}=\frac{(C / D)(\beta)}{(A / D)(\beta)}=(C / A)(\beta) .
$$

If $\beta$ is a root of $B$ of multiplicity $r$ then we may differentiate repeatedly to obtain $r$ linearly independent equations. If we label the distinct roots of $B / D$ as $\beta_{i}$, their multiplicities as $r_{i}$, and for brevity let $n=b-d-1$ then the full system of equations is

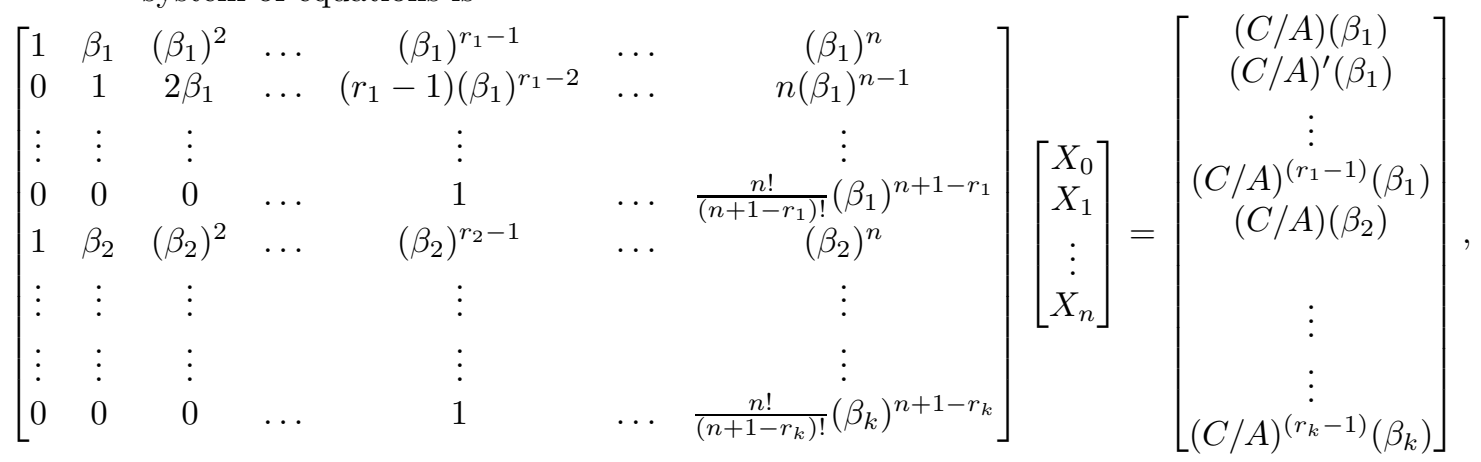

The $(n+1) \times(n+1)$ coefficient matrix on the left is called the confluent Vandermonde matrix at the roots of $B / D$, and we shall denote it $V(B / D)$. We shall denote the vector on the right by $h(B / D, C / A)$. A confluent Vandermonde matrix is always nonsingular [Kal84], therefore there is a unique solution to this system.

Having found $\mathbf{X}$, we may find the corresponding $\mathbf{Y}$ from $B \mathbf{Y}=A \mathbf{X}-C$. To address the final part of the lemma, if $c<a+b-d$, then the degree of the right-hand side above is degree at most $a+b-d-1$, whence the degree of $\mathbf{Y}$ is degree at most $a-d-1$.

Lemma 4.4. Suppose that $(X, Y)$ is a solution to equation (4.1). Further suppose that $A, B$ and $C$ are members of $\mathcal{P}_{\mathbb{R}}^{a}, \mathcal{P}_{\mathbb{R}}^{b}$ and $\mathcal{P}_{\mathbb{R}}^{c}$ respectively, and that $\operatorname{deg} X \leq c-a$. Then there exists a solution to the equation belonging to $\mathcal{P}_{\mathbb{R}}^{c-a} \times$ $\mathcal{P}_{\mathbb{R}}^{c-b}$. Moreover, if $c<a+b-d$ then the minimal solution $(\mathbf{X}, \mathbf{Y})$ itself belongs to $\mathcal{P}_{\mathbb{R}}^{c-a} \times \mathcal{P}_{\mathbb{R}}^{c-b}$.

Proof. Suppose that the equation $A X-B Y=C$ has a solution $(X, Y)$. By applying the involution of $\mathcal{P}_{\mathbb{R}}^{c}$ to (4.1) we see that

$$
A(\zeta) \bar{\zeta}^{c-a} \bar{X}\left(\zeta^{-1}\right)-B(\zeta) \bar{\zeta}^{c-b} \bar{Y}\left(\zeta^{-1}\right)=C(\zeta) .
$$


By assumption, the degree of $X$ is at most $c-a$ so $\bar{\zeta}^{c-a} \bar{X}\left(\zeta^{-1}\right)$ is polynomial. From $A X-B Y=C$ it follows that $Y$ is degree at most $c-b$ and hence $\bar{\zeta}^{c-b} \bar{Y}\left(\zeta^{-1}\right)$ is a polynomial too. By averaging equation (4.5) with the original equation (4.1), we arrive at the following,

$$
A \frac{1}{2}\left(X+\rho_{c-a}^{*} X\right)-B \frac{1}{2}\left(Y+\rho_{c-b}^{*} Y\right)=C,
$$

yielding a real solution as claimed.

In the case that we also have $c<a+b-d$, by subtracting (4.5) from the original equation (4.1), we arrive at the following equation for the 'imaginary parts'

$$
(A / D)\left(\mathbf{X}-\rho_{c-a}^{*} \mathbf{X}\right)-(B / D)\left(\mathbf{Y}-\rho_{c-b}^{*} \mathbf{Y}\right)=0 .
$$

We see therefore that $B / D$ must divide $\mathbf{X}-\rho_{c-a}^{*} \mathbf{X}$, but the former is degree $b-d$ whereas the latter is at most degree $d-b-1$. It follows therefore that $\mathbf{X}=\rho_{c-a}^{*} \mathbf{X}$ and so $\mathbf{X} \in \mathcal{P}_{\mathbb{R}}^{c-a}$. Similarly we have that $\mathbf{Y} \in \mathcal{P}_{\mathbb{R}}^{c-b}$.

Lemma 4.6. Suppose that the polynomials $A, B, C$ are members of $\mathcal{P}_{\mathbb{R}}^{a}, \mathcal{P}_{\mathbb{R}}^{b}, \mathcal{P}_{\mathbb{R}}^{c}$ respectively. If $c \geq a+b-d$, then the space of solutions $(X, Y) \in \mathcal{P}_{\mathbb{R}}^{c-a} \times \mathcal{P}_{\mathbb{R}}^{c-b}$ to equation (4.1) is

$$
\left\{(\mathbf{X}+U(B / D), \mathbf{Y}+U(A / D)) \mid U \in \mathcal{P}_{R}^{c-a-b+d}\right\}
$$

where $(\mathbf{X}, \mathbf{Y})$ is the minimal solution to (4.1) given in Lemma 4.2.

Proof. First suppose that $(Z, W)$ is a solution to the related equation $A Z-$ $B W=0$. After removing the common factor $D$, it must be that $B / D$ divides $Z$. For some polynomial $U$, let $Z=(B / D) U$. Then

$$
(A / D)(B / D) U-(B / D) W=0
$$

from which we conclude that $W=(A / D) U$. If $Z$ and $W$ are real, so too is the quotient $U$.

Return now to the equation $A X-B Y=C$ and suppose then that $(\mathbf{X}, \mathbf{Y})$ the minimal solution given by Lemma 4.2. Because $c-a \geq b-d>\operatorname{deg} \mathbf{X}$, by Lemma 4.4 this is a real solution in $\mathcal{P}_{R}^{c-a} \times \mathcal{P}_{R}^{c-b}$.

If $(X, Y)$ is any other real solution, then $A(X-\mathbf{X})-B(Y-\mathbf{Y})=0$. Therefore $X-\mathbf{X}=(B / D) U$ and $Y-\mathbf{Y}=(A / D) U$ for some real polynomial $U$ in $\mathcal{P}_{\mathbb{R}}^{c-a-b+d}$. Conversely, given any solution $(X, Y)$, it is clear that $(X+$ $(B / D) U, Y+(A / D) U)$ is again a real solution for any real polynomial $U$. Thus polynomials of this form are exactly the desired solutions to $A X-B Y=C$. 


\section{The Tangent Space to $\mathcal{M}_{g}$}

In Section 3, we elucidated several necessary properties of an infinitesimal deformation of spectral triples. Now we turn our attention to the converse; in Lemmata 5.6 and 5.7 we give conditions under which it is possible to solve (3.3) and (3.14) and thereby find an infinitesimal deformation. Firstly, we examine whether it is possible to construct polynomials $c^{i}$ that factor as per (3.12) and solve (3.14) for a given $\tilde{Q}$, and whether this construction is unique. Secondly, we shall insert the polynomials $c^{i}$ into the right hand side of (3.3) and solve it to recover $\dot{b}^{i}$ and $\dot{P}$.

For each equation, the main obstacle to the existence of a solution is common factors among the polynomials $\left(P, b^{1}, b^{2}\right)$. If there are too many common factors (for example if $\operatorname{gcd}\left(b^{1}, b^{2}\right)$ has degree greater than two), then it will not be possible to deform the spectral data. Even when it is possible to deform, the form of the solution of (3.14) is dependent on those common factors. Thus we will need to divide our approach into several cases according to the common roots of $b^{1}$ and $b^{2}$, labelled in the following table. Refer to Figure 3.1 for the definitions of $F$ and $G$.

\begin{tabular}{|c|c|c|c|}
\hline Case & $P_{0}$ & $\operatorname{deg} F$ & $\operatorname{deg} G$ \\
\hline \hline (a) & \multirow{4}{*}{$P_{0} \neq 0$} & 0 & 0 \\
(b) & 0 & 1,2 \\
(c) & & 2 & 0 \\
& & $F G \in \mathcal{P}_{\mathbb{R}}^{k}, k>2$ \\
\hline
\end{tabular}

Recall our standing assumptions that the spectral curve is nonsingular and that it is not branched over $\zeta=0$, ie that the spectral triple is from a nonconformal harmonic map. This is equivalent to $P_{0} \neq 0$. These four cases are disjoint and exhaustive for nonconformal spectral triples.

Definition 5.1. We denote the points of case (a) in $\mathcal{U}$ as $\mathcal{U}_{a}$, and likewise for the other cases. Let $\mathcal{U}_{a b}=\mathcal{U}_{a} \cup \mathcal{U}_{b}$. We may equivalently characterise $\mathcal{U}_{a b}$ as the set

$$
\mathcal{U}_{a b}=\left\{\left(P, b^{1}, b^{2}\right) \in \mathcal{U} \mid \operatorname{gcd}\left(P, b^{1}, b^{2}\right)=1 \text { and } \operatorname{gcd}\left(b^{1}, b^{2}\right) \in \mathcal{P}_{\mathbb{R}}^{l} \text { for } l \leq 2\right\},
$$

which is open and dense in $\mathcal{U}$.

The remainder of the section proves that $\mathcal{M}_{g} \cap \mathcal{U}_{a b}$ is a manifold. We have seen that the spectral triples in case (d) do not admit any deformations. In general, points of $\mathcal{M}_{g}$ where case (c) holds are singularities of a deformation [HKS16] and are not considered further in this paper.

Before we proceed to solving equations, there is a short digression we must make. In case (a) given an arbitrary quadratic polynomial $Q$ it will not always 
be possible to solve (3.14) for polynomials $\tilde{c}^{i}(\zeta)$ corresponding to an infinitesimal deformation $\left(\dot{P}, \dot{b}^{1}, \dot{b}^{2}\right)$. To see why, consider the linear system of equations in the coefficients of $\tilde{c}^{2}$ that arises from evaluating (3.14) at the roots of $\tilde{b}^{2}$,

$$
V\left(\tilde{b}^{2}\right)\left[\begin{array}{c}
\tilde{c}_{0}^{2} \\
\vdots \\
\tilde{c}_{n}^{2}
\end{array}\right]=h\left(\tilde{b}^{2}, \frac{Q \tilde{P}}{\tilde{b}^{1}}\right),
$$

where $V$ is the confluent Vandermonde matrix defined by (4.3). We know that the degree of the unique minimal solution could be as high as

$$
n:=\operatorname{deg} \tilde{b}^{2}-1=g+2-d_{2} .
$$

But the degree of $\tilde{c}^{2}$ is $g+1-d_{2}=n-1$. Thus we must introduce a restriction on our choice of $Q$ so that a solution to (3.14) of the correct degree exists. We will express this restriction in terms of the vanishing of a function $R$.

Definition 5.3. Recall the confluent Vandermonde matrix $V(B)$ and vector $h(B, C / A)$ defined in (4.3). We define the function $R$ to be

$$
\begin{aligned}
R: \mathcal{U}_{a} \times \mathcal{P}_{\mathbb{R}}^{2} & \rightarrow \mathbb{C} \\
\left(\left(P, b^{1}, b^{2}\right), Q\right) & \mapsto \text { the last entry of }\left[V\left(\tilde{b}^{2}\right)\right]^{-1} h\left(\tilde{b}^{2}, \frac{Q \tilde{P}}{\tilde{b}^{1}}\right) .
\end{aligned}
$$

When the point of $\mathcal{U}_{a}$ is understood, we shall abbreviate this to $R(Q)$.

This function $R$ is simply the function that gives the value of the degree $n$ coefficient of $\tilde{c}^{2}$; the condition that $R\left(\left(P, b^{1}, b^{2}\right), Q\right)=0$ is equivalent to the condition that there is a solution $\tilde{c}^{2}$ to (3.14) of degree $n-1$ or less. Likewise, evaluating (3.14) at the roots of $\tilde{b}^{1}$ leads to a solution $\tilde{c}^{1}$ of degree $g+2-d_{1}$. From the highest order term of (3.14), if $\tilde{c}^{2}$ is degree $n-1$ or lower, then $\tilde{c}^{1}$ will be degree $g+1-d_{1}$ or lower without any further restrictions on $Q$.

In case (b), the degree of $\tilde{b}^{2}$ is

$$
g+3-\left(d_{2}+d_{F}+d_{G}\right) \leq g+2-d_{2},
$$

as $G$ will be nontrivial. This means that the minimal solution to (3.14), which has degree strictly less than $\operatorname{deg} \tilde{b}^{2}$, will have the correct degree without needing to impose any extra conditions on $Q$.

It is important to note that $R$ is a linear function in the coefficients of $Q$. $R$ satisfies the following reality type condition and thus at any point of $\mathcal{U}_{a}$ there is a real 2-plane of polynomials $Q \in \mathcal{P}_{\mathbb{R}}^{2}$ that satisfy $R(Q)=0$.

Lemma 5.4. At every point $\left(P, b^{1}, b^{2}\right)$ of $\mathcal{U}_{a}, R\left(\left(P, b^{1}, b^{2}\right), Q\right)$ satisfies the relation

$$
\bar{R}=(-1)^{n}\left(\prod_{i=1}^{n+1} \beta_{i}\right) R,
$$


where $\beta_{i}$ are the $n+1=g+3-d_{2}$ roots of $\tilde{b}^{2}$, counted with multiplicity.

Proof. We shall demonstrate this property first at points where the roots of $\tilde{b}^{2}$ are distinct. Let $\tilde{b}^{2}$ have $n+1=g+3-d_{2}$ distinct roots $\beta_{i}$. In this case, the explicit form of the solution to the linear system of equations (5.2) is elegant. Consider the Lagrange polynomials at the roots of $\tilde{b}^{2}$,

$$
L_{i}(\zeta):=\prod_{j \neq i} \frac{\zeta-\beta_{j}}{\beta_{i}-\beta_{j}} .
$$

Each of these polynomials is degree $n$ and has the property that $L_{i}\left(\beta_{j}\right)=\delta_{i j}$. The unique polynomial of degree at most $n$ solving the linear system is

$$
\tilde{c}^{2}(\zeta)=\sum_{i=1}^{n+1}\left(\frac{Q \tilde{P}}{\tilde{b}^{1}}\right)\left(\beta_{i}\right) L_{i}(\zeta),
$$

and in particular the highest coefficient is $R$,

$$
R=\sum_{i=1}^{n+1}\left(\frac{Q \tilde{P}}{\tilde{b}^{1}}\right)\left(\beta_{i}\right) \prod_{j \neq i}\left(\beta_{i}-\beta_{j}\right)^{-1} .
$$

Because $\tilde{b}^{2}$ is a real polynomial, its set of roots is invariant under $\zeta \mapsto \bar{\zeta}^{-1}$. This creates an involution on the set of roots. Let $\tau$ be the involution on the integers $\{1,2, \ldots, n+1\}$ such that $\beta_{\tau(i)}=\bar{\beta}_{i}^{-1}$. We compute that

$$
\begin{aligned}
\bar{R} & =\sum_{i=1}^{n+1}\left[\left(\frac{Q \tilde{P}}{\tilde{b}^{1}}\right)\left(\beta_{\tau(i)}\right) \bar{\beta}_{i}^{n-1}\right]\left[\prod_{j \neq i} \bar{\beta}_{i}^{-1} \bar{\beta}_{j}^{-1}\left(\beta_{\tau(j)}-\beta_{\tau(i)}\right)^{-1}\right] \\
& =(-1)^{n}\left(\prod_{j=1}^{n+1} \beta_{j}\right) R .
\end{aligned}
$$

To complete the proof, we must extend the argument to points $p=\left(P, b^{1}, b^{2}\right)$ where the roots of $\tilde{b}^{2}$ are not distinct. Suppose that we are at a point $p=$ $\left(P, b^{1}, b^{2}\right)$ of $\mathcal{U}_{a}$ where $\tilde{b}^{2}$ has a double root $\beta$. Considering the subvariety of $\mathcal{U}_{a}$ where $F^{2}=\operatorname{gcd}\left(P, b^{2}\right)$ is fixed, we may find a sequence of points $p_{k}$ in this subvariety converging to $p$ with the property that the roots of each polynomial $\tilde{b}^{2}\left(p_{k}\right)$ are distinct. Let us label the two simple roots of $\tilde{b}^{2}\left(p_{k}\right)$ that coalesce at $p$ to form the double root $\beta$ as $\beta_{1}(k), \beta_{2}(k)$.

Consider the corresponding rows of the Vandermonde matrix $V\left(\tilde{b}^{2}\left(p_{k}\right)\right)$. Performing elementary row operations does not change the solution to this system, and so we may subtract the one row from the other and scale it by $\left(\beta_{2}-\beta_{1}\right)^{-1}$. 
This gives

$$
\left[\begin{array}{ccccc}
1 & \beta_{1} & \left(\beta_{1}\right)^{2} & \ldots & \left(\beta_{1}\right)^{n} \\
0 & 1 & \beta_{2}+\beta_{1} & \ldots & \sum_{j=0}^{n-1}\left(\beta_{1}\right)^{j}\left(\beta_{2}\right)^{n-1-j} \\
& & & \vdots &
\end{array}\right]\left[\begin{array}{c}
\tilde{c}_{0}^{2} \\
\vdots \\
\tilde{c}_{n}^{2}
\end{array}\right]=\left[\begin{array}{c}
\left(\frac{Q \tilde{P}}{b^{1}}\right)\left(\beta_{1}\right) \\
\left(\beta_{2}-\beta_{1}\right)^{-1}\left[\left(\frac{Q \tilde{P}}{\tilde{b}^{1}}\right)\left(\beta_{2}\right)-\left(\frac{Q \tilde{P}}{\tilde{b}^{1}}\right)\left(\beta_{1}\right)\right] \\
\vdots
\end{array}\right] .
$$

The limit of the above as $k \rightarrow \infty$ is precisely the confluent Vandermonde matrix at $\tilde{b}^{2}(p)$. The calculation for higher order roots is similar. If $\tilde{b}^{2}$ has more than one higher order root, then we may perform this operation concurrently for each of them.

Combining these row operations with the fact that inversion of a matrix is continuous and that the roots of a polynomial are continuous functions of coefficients [Whi72, Theorem V.4A], this shows that limit of the solutions $\tilde{c}^{2}\left(p_{k}\right)$ is just the solution $\tilde{c}^{2}(p)$. In particular, the last component of $\tilde{c}^{2}(p)$ is $R(p, Q)$, and so $\lim _{k \rightarrow \infty} R\left(p_{k}, Q\right)=R(p, Q)$. Hence we have established (5.5) at all points of $\mathcal{U}_{a}$.

At this point we are ready to solve equations (3.3) and (3.14) in both of the cases (a) and (b). At the end of that process, we will have constructed a tangent vector $\left(\dot{P}, \dot{b}^{1}, \dot{b}^{2}\right)$ to the space of spectral triples $\mathcal{M}_{g}$.

Lemma 5.6 (Case (a)). Take a spectral triple $\left(P, b^{1}, b^{2}\right) \in \mathcal{M}_{g}$ associated with a nonconformal harmonic map, with a nonsingular spectral curve given by $\eta^{2}=P$ of genus $g$. Suppose that $\operatorname{gcd}\left(b^{1}, b^{2}\right)=1$. Then for every polynomial $Q \in \mathcal{P}_{\mathbb{R}}^{2}$ with $R(Q)=0$, there exist unique real polynomials $c^{i} \in \mathcal{P}_{\mathbb{R}}^{g+1}$ that factor as per (3.12) and satisfy (3.14). Further, for each such pair $\left(c^{1}, c^{2}\right)$, there is a unique vector $\left(\dot{P}, \dot{b}^{1}, \dot{b}^{2}\right) \in \mathcal{P}_{\mathbb{R}}^{2 g+2} \times \mathcal{P}_{\mathbb{R}}^{g+3} \times \mathcal{P}_{\mathbb{R}}^{g+3}$ that satisfies (3.3) and (3.9). It is therefore a tangent vector to the space of spectral triples $\mathcal{M}_{g}$.

Proof. Let us first begin with (3.14). By Lemma 4.2 there is a unique solution $\left(\tilde{\mathbf{c}}^{1}, \tilde{\mathbf{c}}^{2}\right)$ to this equation of degree at most $\left(g+2-d_{1}, g+2-d_{2}\right)$, where $d_{i}=$ $\operatorname{deg} F^{i}$. We note that the leading coefficient $\tilde{\mathbf{c}}_{g+2-d_{2}}^{2}$ is $R(Q)$ by definition, which by assumption is zero. Examining the highest order of (3.14), if $\tilde{\mathbf{c}}_{g+2-d_{2}}^{2}$ vanishes, so too must $\tilde{\mathbf{c}}_{g+2-d_{1}}^{1}$. Multiplying (3.14) through by $F^{1} F^{2}$, we arrive at unique polynomials $c^{i}=F^{i} \tilde{\mathbf{c}}^{i}$ with the factors required by (3.12). Both of these polynomials are degree at most $g+1$. By Lemma 4.4 these are real polynomials. We similarly define $\hat{c}^{i}=\left(\zeta^{2}-1\right) c^{i}$.

Next we must solve (3.3). To see that Lemma 4.4 will apply, for any $f \in \mathcal{P}_{\mathbb{R}}^{k}$ we compute that

$$
\rho_{k}^{*}\left(\zeta f^{\prime}\right)=\zeta^{k-1} \bar{f}^{\prime}\left(\zeta^{-1}\right)=k f(\zeta)-\zeta f^{\prime}(\zeta)
$$

From this it follows that the right hand side of (3.3) is real for all real polynomials $P$ and imaginary polynomials $\hat{c}^{i}$, not just when these polynomials arise from a deformation. It can be solved using a Vandermonde matrix, if $\tilde{b}^{i}$ is 
non-vanishing at the roots of $F^{j} \tilde{P}$ and vice versa. But by definition they are coprime.

The two equations for $i=1,2$ may give different solutions for $\dot{P}$, and indeed in general they do. However, we will show that there is a common solution to both. Let a solution to each equation (3.3) be $\left(\dot{\mathbf{P}}^{1}, \dot{\mathbf{b}}^{1}\right)$ and $\left(\dot{\mathbf{P}}^{2}, \dot{\mathbf{b}}^{2}\right)$. From Lemma 4.6 the sets of solutions of degree $(2 g+2, g+3)$ are

$$
\left\{\left(\dot{\mathbf{P}}^{1}+2 r F^{2} \tilde{P}, \dot{\mathbf{b}}^{1}+r \tilde{b}^{1}\right) \mid r \in \mathcal{P}_{\mathbb{R}}^{d_{1}}\right\},
$$

and

$$
\left\{\left(\dot{\mathbf{P}}^{2}+2 s F^{1} \tilde{P}, \dot{\mathbf{b}}^{2}+s \tilde{b}^{2}\right) \mid s \in \mathcal{P}_{\mathbb{R}}^{d_{2}}\right\},
$$

respectively. First note that every element of both of these sets take the same value at any root $\alpha$ of $\tilde{P}$. This follows from

$$
\dot{P}^{1}(\alpha)=\alpha\left(\alpha^{2}-1\right) P^{\prime}(\alpha) \frac{\tilde{c}^{1}(\alpha)}{\tilde{b}^{1}(\alpha)}=\alpha\left(\alpha^{2}-1\right) P^{\prime}(\alpha) \frac{\tilde{c}^{2}(\alpha)}{\tilde{b}^{2}(\alpha)}=\dot{P}^{2}(\alpha),
$$

where we have used (3.3) and (3.14) evaluated at $\alpha$. At the $d_{1}$ roots of $F^{1}$, we see that every solution $\dot{P}^{2}$ takes the same value. Let $\beta$ be such a root, then

$$
\dot{P}^{2}(\beta)=\dot{\mathbf{P}}^{2}(\beta)+2 s(\beta) F^{1}(\beta) \tilde{P}(\beta)=\dot{\mathbf{P}}^{2}(\beta)=\beta\left(\beta^{2}-1\right) P^{\prime}(\beta) \frac{\tilde{c}^{2}(\beta)}{\tilde{b}^{2}(\beta)},
$$

However, the other solutions have different values at $\beta$, and this provides the following constraint on the choice of $r$ :

$$
\dot{\mathbf{P}}^{1}(\beta)+2 r(\beta) F^{2}(\beta) \tilde{P}(\beta)=\beta\left(\beta^{2}-1\right) P^{\prime}(\beta) \frac{\tilde{c}^{2}(\beta)}{\tilde{b}^{2}(\beta)} .
$$

This constraint is nontrivial because $\beta$ is not a root of $\tilde{P}$ or $F^{2}$ by the assumption of the nonsingularity of the spectral curve. As $F^{1}$ has $d_{1}$ distinct roots, there are $d_{1}$ constraints.

Likewise, at the $d_{2}$ roots of $F^{2}$, we acquire constraints on the choice of $s$. It is always possible to meet these constraints (because, for example, the degree of $s$ is $d_{2}$ and there are only $d_{2}$ roots of $F^{2}$ ), so we see that there is a common solution $\left(\dot{\mathbf{P}}, \dot{\mathbf{b}}^{1}, \dot{\mathbf{b}}^{2}\right)$ to $(3.3)$.

This solution is still not unique; there remains one real parameter. For any real number $s$, we have solutions to (3.3) of the form

$$
\dot{P}=\dot{\mathbf{P}}+2 s P, \quad \dot{b}^{i}=\dot{\mathbf{b}}^{i}+s b^{i}
$$

However, this freedom rescales $P$. We have chosen a preferred scaling of $P$, so our choice of $s$ is determined. 
Finally there is another necessary condition that must be satisfied by our solution $\left(\dot{P}, \dot{b}^{1}, \dot{b}^{2}\right)$. We must satisfy $(3.9)$, so that $(2.4)$ holds along the path. But this condition is satisfied automatically. Observe

$$
\begin{aligned}
\dot{P}_{1} b_{0}^{i}+P_{1} \dot{b}_{0}^{i}-2\left(\dot{P}_{0} b_{1}^{i}+P_{0} \dot{b}_{1}^{i}\right) & =3\left(P_{1} \dot{b}_{0}^{i}-\dot{P}_{0} b_{1}^{i}+P_{1} \hat{c}_{0}\right) \\
& =\frac{3}{P_{0}}\left(P_{0} P_{1} \dot{b}_{0}^{i}-P_{0} \dot{P}_{0} b_{1}^{i}+P_{1}\left(\frac{1}{2} \dot{P}_{0} b_{0}^{i}-P_{0} \dot{b_{0}^{i}}\right)\right) \\
& =\frac{3 \dot{P}_{0}}{P_{0}}\left(-P_{0} b_{1}^{i}+\frac{1}{2} P_{1} b_{0}^{i}\right)=0
\end{aligned}
$$

The substitution in the first line comes from the $\zeta^{1}$ terms of (3.8), the second line from the constant terms of (3.8) and the last line comes from the fact that the quantity in the bracket is exactly the residue at $\zeta=0$, which is zero by the assumption that $\left(P, b^{1}, b^{2}\right)$ lies in $\mathcal{M}_{g}$, the space of spectral data.

Hence $\left(\dot{P}, \dot{b}^{1}, \dot{b}^{2}\right)$ is a tangent vector to $\mathcal{M}_{g}$ at $\left(P, b^{1}, b^{2}\right)$.

Lemma 5.7 (Case (b)). Take a spectral triple $\left(P, b^{1}, b^{2}\right) \in \mathcal{M}_{g}$ associated with a nonconformal harmonic map, with a nonsingular spectral curve given by $\eta^{2}=P$ of genus $g$. Suppose that $G=\operatorname{gcd}\left(b^{1}, b^{2}\right)$ is a non-constant real polynomial that does not divide $P$. If $G$ lies in $\mathcal{P}_{\mathbb{R}}^{1}$ then for every polynomial $\tilde{Q} \in \mathcal{P}_{\mathbb{R}}^{1}$, or if $G$ lies in $\mathcal{P}_{\mathbb{R}}^{2}$ then for every pair of real numbers $(\tilde{Q}, r)$, there exist unique real polynomials $c^{i} \in \mathcal{P}_{\mathbb{R}}^{g+1}$ that factor as per (3.12) and satisfy (3.14). Further, for each such pair $\left(c^{1}, c^{2}\right)$, there is a unique vector $\left(\dot{P}, \dot{b}^{1}, \dot{b}^{2}\right) \in \mathcal{P}_{\mathbb{R}}^{2 g+2} \times \mathcal{P}_{\mathbb{R}}^{g+3} \times \mathcal{P}_{\mathbb{R}}^{g+3}$ that satisfies (3.3) and (3.9).

Proof. The proof of this lemma is similar to that of 5.6. We proceed by first solving (3.14) and using the resulting pair $\left(c^{1}, c^{2}\right)$ as inputs to solve (3.3). Regardless of the degree of $G$, which we recall is denoted $d_{G}$, we must set $Q=G \tilde{Q}$. Equation (3.14) reads

$$
\tilde{b}^{1} \tilde{c}^{2}-\tilde{b}^{2} \tilde{c}^{1}=\tilde{Q} \tilde{P} .
$$

There is a unique solution to this equation $\left(\tilde{\mathbf{c}}^{1}, \tilde{\mathbf{c}}^{2}\right)$ of degree at most $(g+2-$ $d_{1}-d_{G}, g+2-d_{2}-d_{G}$ ). If $G$ is linear, multiplying the unique solution of degree at most $\left(g+1-d_{1}, g+1-d_{2}\right)$ by $F^{1}$ and $F^{2}$ respectively gives the desired pair $\left(c^{1}, c^{2}\right)$. But if $G$ is quadratic the space of solutions to (3.14) is

$$
\left\{\left(\tilde{\mathbf{c}}^{1}+r \tilde{b}^{1}, \tilde{\mathbf{c}}^{1}+r \tilde{b}^{2}\right) \mid r \in \mathbb{R}\right\} .
$$

Hence in that case, for every $r \in \mathbb{R}$ there is a unique pair $\left(c^{1}, c^{2}\right)$ that factors as required and solves (3.14). In both cases it was not necessary to have a extraneous condition such as $R(Q)=0$, but the choice of $Q$ was restricted by $Q=G \tilde{Q}$.

Next we must solve (3.3), but the proof in Lemma 5.6 applies essentially without modification. It has a solution because $\operatorname{gcd}\left(F^{j} \tilde{P}, G \tilde{b}^{i}\right)=1$. Analysis at the roots 
of $F^{1} F^{2} \tilde{P}$ shows that there is a common solution $\left(\dot{\mathbf{P}}, \dot{\mathbf{b}}^{1}, \dot{\mathbf{b}}^{2}\right)$. Again, a choice of scaling of $P$ forces a unique solution. This solution also satisfies (3.9). Hence it is a tangent vector to $\mathcal{M}_{g}$ at $\left(b^{1}, b^{2}, P\right)$.

Both lemmata above tell essentially the same story, that there there is a choice of two real parameters each giving a unique tangent vector. Conversely, given any tangent vector $\left(\dot{P}, \dot{b}^{1}, \dot{b}^{2}\right)$ to $\mathcal{M}_{g}$ there is a unique pair of polynomials $\left(\hat{c}^{1}, \hat{c}^{2}\right)$, as shown in Lemma 3.4, and thus a unique polynomial $Q$ from (3.8). Hence this pairing between parameters and tangent vectors is bijective, and we may identify the tangent space to $\mathcal{M}_{g}$ with these two real parameters. This suggests that $\mathcal{M}_{g} \cap \mathcal{U}$ itself is a surface.

Theorem 5.8. The open subset $\mathcal{M}_{g} \cap \mathcal{U}_{a b}$ of the space of spectral triples $\mathcal{M}_{g}$ is a two dimensional manifold.

Proof. Recall Definition 5.1 of $\mathcal{U}_{a b}$ as the open set whose points correspond to cases (a) or (b) and note this is an open set. At any point $p \in \mathcal{M}_{g} \cap \mathcal{U}_{a b}$, take a simply connected open neighbourhood $\mathcal{V} \subset \mathcal{U}$. On this neighbourhood, define the map $\Psi: \mathcal{V} \rightarrow \mathbb{R}^{4 g+9}$ by

$$
\begin{aligned}
\Psi\left(P, b^{2}, b^{2}\right)= & \left(\int_{A_{1}} \Theta^{1}, \ldots, \int_{A_{g}} \Theta^{1}, \int_{B_{1}} \Theta^{1}, \ldots, \int_{B_{g}} \Theta^{1},\right. \\
& \int_{A_{1}} \Theta^{2}, \ldots, \int_{A_{g}} \Theta^{2}, \int_{B_{1}} \Theta^{2}, \ldots, \int_{B_{g}} \Theta^{2}, \\
& \int_{\gamma_{+}} \Theta^{1}, \int_{\gamma_{-}} \Theta^{1}, \int_{\gamma_{+}} \Theta^{2}, \int_{\gamma_{-}} \Theta^{2}, \\
& \left.P_{1} b_{0}^{1}-2 P_{0} b_{1}^{1}, P_{1} b_{0}^{2}-2 P_{0} b_{1}^{2},\left(P_{0}\right)^{-1} \prod_{k}\left(-\alpha_{k}\right)\right)
\end{aligned}
$$

where $A_{i}, B_{i}$ are the real and imaginary periods of $\Sigma, \gamma_{+}$and $\gamma_{-}$are paths in $\Sigma$ between the points over $\zeta=1$ and $\zeta=-1$, and $\alpha_{k}$ are the roots of $P$ inside the unit circle. Because $\mathcal{V}$ is simply connected, the choice of paths $\left\{A_{k}\right\},\left\{B_{k}\right\}, \gamma_{+}, \gamma_{-}$may be made smoothly and consistently. The components of $\Psi$ are the conditions that spectral data must satisfy. In particular, the first $4 g$ components of $\Psi$ are the periods of the differentials, the next four components are the integrals in the closing conditions (P.9), followed by the conditions to have no residues (2.5) and the last component of $\Psi$ is our preferred scaling of the spectral curve.

Hence $\mathcal{M}_{g} \cap \mathcal{V}$ is contained in the level sets of $\Psi$,

$$
\begin{gathered}
\mathcal{M}_{g} \cap \mathcal{V} \subset \Psi^{-1}(0, \ldots, 0,2 \pi i \mathbb{Z}, \ldots, 2 \pi i \mathbb{Z}, 0, \ldots, 0,2 \pi i \mathbb{Z}, \ldots, 2 \pi i \mathbb{Z} \\
2 \pi i \mathbb{Z}, 2 \pi i \mathbb{Z}, 2 \pi i \mathbb{Z}, 2 \pi i \mathbb{Z}, 0,0,1)
\end{gathered}
$$

The point $p$ of $\mathcal{M}_{g} \cap \mathcal{V}$ falls under either Lemma 5.6 or 5.7. In both cases we computed that the kernel of $d \Psi_{p}$ is two dimensional. The differential of $\Psi$ 
is a map from $\mathbb{R}^{4 g+11}$ to $\mathbb{R}^{4 g+9}=\mathbb{R}^{4 g+4+2+2+1}$, and so is full rank at every such point $p$. Therefore by the Implicit Function Theorem $\mathcal{M}_{g} \cap \mathcal{U}_{a b}$ is a two dimensional manifold.

\section{Conformal Harmonic Maps}

From equation (3.11) onwards, we made the assumption that the spectral triple came from a nonconformal harmonic map. However, conformal harmonic maps are of particular interest because they are minimal immersions. We show in this section, under mild assumptions, that the points of $\mathcal{M}_{g}$ corresponding to conformal maps are also smooth points of dimension two.

Let us return to the discussion following (3.11), this time assuming that a point of $\mathcal{M}_{g}$ corresponds to a conformal map. We know that $P_{0}(t), b_{0}^{1}(t)$ and $b_{0}^{2}(t)$ all vanish at $t=0$. Thus $F=\operatorname{gcd}\left(P, b^{1}, b^{2}\right)$ includes a factor of $\zeta$. It is now $\zeta^{-1} F F^{i}$ that divides $c^{i}$ and so $\zeta^{-1} F G$ divides $Q$, by (3.8). However the residue condition (3.9) in this case simplifies in a way that forces another constraint on $Q$. At $t=0,(3.9)$ becomes

$$
P_{1} \dot{b}_{0}-2 \dot{P}_{0} b_{1}=0
$$

Combining this with the terms of linear degree in equation (3.3) gives

$$
3 P_{1} \hat{c}_{0}^{i}=\dot{P}_{0} b_{1}^{i}-4 \dot{P}_{0} b_{1}^{i}=-3 \dot{P}_{0} b_{1}^{i} .
$$

Substituting this into the linear degree of (3.8), we arrive at

$$
Q_{0}\left(P_{1}\right)^{2}=b_{1}^{1}\left(P_{1} c_{0}^{2}\right)-b_{1}^{2}\left(P_{1} c_{0}^{1}\right)=0 .
$$

A spectral curve must be nonsingular at $\zeta=0$, so if $P_{0}=0$ we can be sure that $P_{1} \neq 0$. Hence $Q_{0}$ must vanish. As $Q$ is a real quadratic polynomial, it must be of the form $Q=Q_{1} \zeta$ for some real number $Q_{1}$. Immediately it follows that if a deformation exists at a point corresponding to a conformal map then $F=\zeta$ and $G=1$, as the polynomials $b^{i}$ are not permitted to have multiple roots at $\zeta=0$. Thus there are two conformal cases, and only (e) can lead to deformations.

\begin{tabular}{|c|c|c|c|}
\hline Case & $P_{0}$ & $\operatorname{deg} F$ & $\operatorname{deg} G$ \\
\hline \hline (e) & \multirow{2}{*}{$P_{0}=0$} & $F=\zeta$ & 0 \\
\cline { 3 - 4 } & & $F G \in \mathcal{P}_{\mathbb{R}}^{k}, k>2$ \\
\hline
\end{tabular}

Without further delay, let us show that the necessary equations can be solved in case (e).

Lemma 6.2 (Case (e)). Take a spectral triple $\left(P, b^{1}, b^{2}\right) \in \mathcal{M}_{g}$ associated with a conformal harmonic map, with a nonsingular spectral curve given by $\eta^{2}=P$ 
of genus $g$. Suppose that $\operatorname{gcd}\left(b^{1}, b^{2}\right)=\zeta$. Then for every pair of real numbers $\left(Q_{1}, r\right)$, there exist unique real polynomials $c^{i} \in \mathcal{P}_{\mathbb{R}}^{g+1}$ that factor as per (3.12) and satisfy (3.14). Further, for each such pair $\left(c^{1}, c^{2}\right)$, there is a unique vector $\left(\dot{P}, \dot{b}^{1}, \dot{b}^{2}\right) \in \mathcal{P}_{\mathbb{R}}^{2 g+2} \times \mathcal{P}_{\mathbb{R}}^{g+3} \times \mathcal{P}_{\mathbb{R}}^{g+3}$ that satisfies (3.3) and (3.9). It is therefore a tangent vector to the space of spectral data $\mathcal{M}_{g}$.

Proof. This is the conformal case, so $P(0)=P_{0}=0$. From (2.5), $b_{0}^{i}=0$ also. We may write therefore that $P=\zeta F^{1} F^{2} \tilde{P}$ and $b^{i}=\zeta F^{i} \tilde{b}^{i}$, where $\tilde{P} \in \mathcal{P}^{2 g-d_{1}-d_{2}}$ and the polynomials $\tilde{b}^{i} \in \mathcal{P}_{\mathbb{R}}^{g+1-d_{i}}$ are coprime.

We have already demonstrated in (6.1) that $\zeta$ necessarily divides $Q$. Thus (3.14) is simply

$$
\tilde{b}^{1} \tilde{c}^{2}-\tilde{b}^{2} \tilde{c}^{1}=\zeta Q_{1} \tilde{P} .
$$

This is similar to the above case where $G$ was quadratic (Lemma 5.7). The space of solutions is

$$
\left\{\left(\tilde{\mathbf{c}}^{1}+r \tilde{b}^{1}, \tilde{\mathbf{c}}^{2}+r \tilde{b}^{2}\right) \mid r \in \mathbb{R}\right\},
$$

where $\left(\tilde{\mathbf{c}}^{1}, \tilde{\mathbf{c}}^{2}\right)$ is the unique solution of degree at most $\left(g+1-d_{1}, g+1-d_{2}\right)$. For every such solution, let $c^{i}=F^{i} \tilde{c}^{i}$ and consider the corresponding (3.3). As before, there is a common solution $\left(\dot{\mathbf{P}}, \dot{\mathbf{b}}^{1}, \dot{\mathbf{b}}^{2}\right)$ for the dotted quantities. The space of solutions is however

$$
\left\{\left(\dot{\mathbf{P}}+2 s F^{1} F^{2} \tilde{P}, \dot{\mathbf{b}}^{1}+s F^{1} \tilde{b}^{1}, \dot{\mathbf{b}}^{2}+s F^{2} \tilde{b}^{2}\right) \mid s \in \mathcal{P}_{\mathbb{R}}^{2}\right\},
$$

and to each choice $(Q, r)$ there are many tangent vectors. However, unlike the cases (a) and (b), equation (3.9) is not automatically satisfied. Let $s=$ $s_{0}+s_{1} \zeta+\bar{s}_{0} \zeta^{2}$. For $i=1$, we see that the condition implies that

$$
2 \dot{\mathbf{P}}_{0} b_{1}^{1}-P_{1} \dot{\mathbf{b}}_{0}^{1}+3 s_{0} P_{1} b_{1}^{1}=0,
$$

which fully determines $s_{0}$. We now show that this solution simultaneously satisfies the condition for $i=2$. Note that (3.3) in the lowest degree reads

$$
\dot{P}_{0} b_{1}^{i}-2 P_{1} \dot{b}_{0}^{i}=-3 P_{1} c_{0}^{i},
$$

and (6.3) in the lowest degree yields

$$
2 b_{1}^{1} \dot{\mathbf{b}}_{\mathbf{0}}^{2}=2 b_{1}^{2} \dot{\mathbf{b}}_{\mathbf{0}}^{1} .
$$

Condition (3.9) for $i=2$ is therefore

$$
b_{1}^{1}\left(2 \dot{\mathbf{P}}_{\mathbf{0}} b_{1}^{2}-P_{1} \dot{\mathbf{b}}_{\mathbf{0}}^{2}+3 s_{0} P_{1} b_{1}^{2}\right)=b_{1}^{2}\left(2 \dot{\mathbf{P}}_{\mathbf{0}} b_{1}^{1}-P_{\mathbf{1}} \dot{\mathbf{b}}_{\mathbf{0}}^{\mathbf{1}}+3 s_{0} P_{1} b_{1}^{1}\right)=0 .
$$

Hence we have demonstrated that the condition holds for $i=2$ also. Having cleared this hurdle, there is still one free parameter. For any $Q_{1}$ and $r$, the 
corresponding tangent vectors that solve (3.3) are

$$
\begin{aligned}
\{(\dot{\mathbf{P}}+ & 2\left(s_{0}+\bar{s}_{0} \zeta^{2}\right) F^{1} F^{2} \tilde{P}+2 s_{1} \zeta F^{1} F^{2} \tilde{P}, \\
& \dot{\mathbf{b}}^{1}+\left(s_{0}+\bar{s}_{0} \zeta^{2}\right) F^{1} \tilde{b}^{1}+s_{1} \zeta F^{1} \tilde{b}^{1}, \\
& \left.\left.\dot{\mathbf{b}}^{2}+\left(s_{0}+\bar{s}_{0} \zeta^{2}\right) F^{2} \tilde{b}^{2}+s_{1} \zeta F^{2} \tilde{b}^{2}\right) \quad \mid s_{1} \in \mathbb{R}\right\} .
\end{aligned}
$$

But our free choice of $s_{1} \in \mathbb{R}$ is only adding multiples of $\left(2 P, b^{1}, b^{2}\right)$, which as in the nonconformal case is a rescaling of the spectral curve, and so also determined uniquely.

From this Lemma there is a result analogous to Theorem 5.8. As in that theorem, we can show that every point of $\mathcal{M}_{g} \cap \mathcal{U}_{e}$ is a smooth point of $\mathcal{M}_{g}$. However, $\mathcal{U}_{e}$ is not an open set, so we cannot say that $\mathcal{M}_{g} \cap \mathcal{U}_{e}$ is a manifold.

Theorem 6.4. The points of $\mathcal{M}_{g} \cap \mathcal{U}_{e}$, corresponding to conformal harmonic maps, are smooth points of $\mathcal{M}_{g}$ of dimension two.

Proof. Is entirely similar to Theorem 5.8.

\section{Consequences}

In this final section we collect some corollaries. First we show that when the genus $g$ of the spectral curve is zero or one, it cannot have singularities and cases (c), (d) and (f) do not occur. Thus $\mathcal{M}_{0}$ and $\mathcal{M}_{1}$ are smooth at every point and therefore are surfaces. Then we comment on why case (c) is difficult. And finally we give an interpretation of $Q$.

Firstly, we can rule out singular spectral curves with genus two or less. In (arithmetic) genus zero, there is one one pair of branch points so singularities are plainly impossible. In higher genus, suppose we have a singular spectral curve $\Sigma$ with normalisation $\tilde{\Sigma}$. Because there can be no singular points on the unit circle, all singular points come in pairs, so the genus of $\Sigma$ and $\tilde{\Sigma}$ differ by at least two. This excludes the possibility of singular spectral curves of genus zero or one. If we add in the fact that at a point of $\tilde{\Sigma}$ that maps to $\Sigma$ with multiplicity $m$, the differentials $\Theta$ and $\tilde{\Theta}$ both have a common zero of order $m-1$, then Lemma 7.1 below shows that no singular spectral curve has a genus zero normalisation. This rules out singular genus two spectral curves also.

To exclude the possibility of the undesirable cases (c), (d) and (f), we need to know if the differentials have a common root. For genus zero this is answered by the following lemma. In [CS16b], a stronger result analogous to 7.1 is proved by examining the degree of $b^{1} / b^{2}$ considered as a function $\mathbb{C P}^{1} \rightarrow \mathbb{C P}^{1}$. 
Lemma 7.1. On a spectral curve of genus zero, differentials satisfying conditions (P.4)-(P.6) with linearly independent principal parts do not have common roots.

Proof. We distinguish between two cases: whether or not the spectral curve is branched over $\zeta=0$. If $\zeta=0$ is a branch point, then we note the following more general proof. Suppose the spectral curve has genus $g$. Then from (2.3) we have that any differential may be written as

$$
a(\zeta) \frac{d \zeta}{\zeta \eta}
$$

for some real polynomial $a$ of degree $g+1$. Any real polynomial is determined up to real scaling by its $g+1$ roots, so if two such differentials have $g+1$ roots in common, then they are linearly dependent over $\mathbb{R}$. Letting $g=0$ shows that two differentials may not share any roots.

In the nonconformal case, instead we consider the specific form of the differentials. Suppose that the spectral curve is branched over $\alpha$ and $\bar{\alpha}^{-1}$ and let $x=-\frac{1}{2} \alpha^{-1}(1+\alpha \bar{\alpha})$. Then a differential $\Theta$ satisfying (P.4)-(P.6) is given by

$$
\Theta=\left(y+x y \zeta+\overline{x y} \zeta^{2}+\bar{y} \zeta^{3}\right) \frac{d \zeta}{\zeta^{2} \eta},
$$

for some a nonzero constant $y$. If $\Theta$ has a root at $\beta$, then

$$
\frac{y}{\bar{y}}=-\beta^{2} \frac{\bar{x}+\beta}{1+x \beta} \text {. }
$$

So any two such differentials with a common root are linearly dependent over $\mathbb{R}$.

We shall now give a similar short proof to show that the differentials of a genus one spectral curve may not have a common root at the branch points, which excludes (c). Suppose that $\Sigma$ is genus one with branch points at $\alpha, \beta, \bar{\alpha}^{-1}, \bar{\beta}^{-1}$, none of which are zero, and that $\operatorname{gcd}\left(P, b^{1}, b^{2}\right)=F$ is quadratic. Without loss of generality, let $F=(\zeta-\alpha)(1-\bar{\alpha} \zeta)$ and $b^{1}=\left(c+d \zeta+\bar{c} \zeta^{2}\right) F$ for some complex number $c$ and real number $d$. Expanding this and applying (2.5) shows that

$$
0=\left(\alpha^{2}(1+\beta \bar{\beta})-\alpha \beta(1+\alpha \bar{\alpha})\right) c+2 \alpha^{2} \beta d .
$$

The coefficient of $c$ above, the bracketed expression, is never zero and so $c$ is determined by $d$. Hence $b^{1}$ is determined up to a real scalar. This demonstrates any two differentials with the same factor $F$ are real linearly dependent, which contradicts (P.7). If the differentials have common roots as per case (d) or (f), then they are forced to be real linearly dependent by the residue condition. 
Thus we have shown that for a spectral curve of genus zero or one, it cannot have singularities and cases (c), (d) and (f) do not occur. Thus $\mathcal{M}_{0}$ and $\mathcal{M}_{1}$ are smooth at every point and therefore are surfaces.

In higher genus, it is possible that there are exist harmonic tori that fall under cases (c), (d), or (f). Cases (d) and (f) do not admit deformations, so could be isolated points of $\mathcal{M}_{g}$, cusps, or some other type of singular point. Case (c) however could be a smooth point of $\mathcal{M}_{g}$. The obstacle to proving a result analogous to Lemma 5.7 is solving equation (3.14), which would read

$$
\tilde{b}^{2} \tilde{c}^{2}-\tilde{b}^{2} \tilde{c}^{1}=\tilde{Q} \tilde{P}
$$

for $\tilde{Q} \in \mathbb{R}$. As the degree of $\tilde{b}^{2}$ is $g+1-d_{2}$, there is a solution such that $\tilde{c}^{2}$ has degree $g-d_{2}$. However, if $\tilde{c}^{2}$ came from a deformation, then we would expect it to have degree $g-1-d_{2}$. This is similar to case (a), where we imposed a condition on $Q$ to ensure the correct degree, namely that $R(Q)=0$. In this case however, the space of allowed $Q$ is already one dimensional. Therefore whether or not an infinitesimal deformation exists depends in a transcendental way on the spectral curve.

We can spell out this dependence explicitly. Given a spectral curve, it determines a plane of differential satisfying conditions (P.4)-(P.8) and therefore determines $F=\operatorname{gcd}\left(P, b^{1}, b^{2}\right)$. The space $\mathcal{M}_{g} \cap \mathcal{U}_{e}$ is the subset of spectral triples where $F$ is quadratic and the plane of differentials contains two linearly independent differentials with integral periods. To show that deformations are always possible in case (c) is equivalent to showing that $R\left(\left(P, b^{1}, b^{2}\right), F\right)$ vanishes on $\mathcal{M}_{g} \cap \mathcal{U}_{e}$.

To close, let us give a geometric interpretation to the polynomial $Q$. The conformal type of the domain of a harmonic tori is given by the ratios of the principal parts of the differentials of its spectral data. Let the conformal type be denoted $\tau$. For a nonconformal harmonic tori we have that $b_{0}^{2}=\tau b_{0}^{1}$. Consideration of the constant terms of (3.3) reveal that

$$
\dot{P}_{0} b_{0}^{i}-2 P_{0} \dot{b}^{i}=2 P_{0} \hat{c}_{0}^{i}
$$

We substitute this into (3.8) to arrive at

$$
Q_{0} P_{0}=b_{0}^{1} \dot{b}_{0}^{2}-\dot{b}_{0}^{1} b_{0}^{2}
$$

Differentiating the relationship $b_{0}^{2}=\tau b_{0}^{1}$ and rearranging the above yields

$$
Q_{0}=\frac{\dot{\tau}}{\tau} \frac{b_{0}^{1} b_{0}^{2}}{P_{0}}
$$

We see therefore that $Q_{0}$ controls the change in the conformal type of the domain of the harmonic map. 


\section{References}

[CS16a] Carberry E and Schmidt M U. The closure of spectral data for constant mean curvature tori in 3. Journal für die reine und angewandte Mathematik (Crelles Journal), 2016(721), 2016.

[CS16b] Carberry E and Schmidt M U. The prevalence of tori amongst constant mean curvature planes in r3. Journal of Geometry and Physics, 106:352-366, 2016.

[FFM80] Flaschka H, Forest M G, and McLaughlin D W. Multiphase averaging and the inverse spectral solution of the Korteweg-de Vries equation. Communications on Pure and Applied Mathematics, 33(6):739-784, 1980.

[GKM $\left.{ }^{+} 95\right]$ Gorsky A, Krichever I, Marshakov A, Mironov A, and Morozov A. Integrability and Seiberg-Witten exact solution. Physics Letters B, 355(3-4):466-474, 1995.

[Gru12] Grushevsky S. The Schottky problem. In Current Developments in Algebraic Geometry, volume 59 of Mathematical Sciences Research Institute Publications, pages 129-164. Cambridge University Press, Cambridge, 2012.

[Hit90] Hitchin N J. Harmonic maps from a 2-torus to the 3-sphere. Journal of Differential Geometry, 31:627-710, 1990.

[HKS16] Hauswirth L, Kilian M, and Schmidt M U. Singularities of Whitham flows for hyperelliptic spectral curves, 2016. Preprint.

[Kal84] Kalman D. The generalized Vandermonde matrix. Mathematics Magazine, 57(1):15-21, 1984.

[Kri95] Krichever I. Algebraic-geometrical methods in the theory of integrable equations and their perturbations. Acta Applicandae Mathematicae, 39(1-3):93-125, 1995.

[KSS15] Kilian M, Schmidt M U, and Schmitt N. Flows of constant mean curvature tori in the 3-sphere: The equivariant case. Journal für die Reine und Angewandte Mathematik (Crelle's Journal), 707:45-86, 2015.

[LL83] Lax P D and Levermore C D. The small dispersion limit of the Korteweg-de Vries equation. I. Communications on Pure and Applied Mathematics, 36(10):253-290, 1983.

[Mir95] Miranda R. Algebraic Curves and Riemann Surfaces, volume 5 of Graduate Studies in Mathematics. American Mathematical Society, Providence, RI, 1995. 
[Shi86] Shiota T. Characterization of Jacobian varieties in terms of soliton equations. Inventiones Mathematicae, 83(2):333-382, 1986.

[Whi72] Whitney H. Complex Analytic Varieties. Addison-Wesley Publishing Co., Reading, Massachusetts, 1972. 